Corrigendum

\title{
Corrigendum to "Handedness Side and Magnetization Transfer Ratio in the Primary Sensorimotor Cortex Central Sulcus"
}

\author{
Mario Mascalchi, ${ }^{1}$ Stefano Ciulli, ${ }^{1}$ Andrea Bianchi, ${ }^{1}$ Chiara Marzi, ${ }^{2}$ Stefano Orsolini, ${ }^{2}$ \\ Gioele Gavazzi, ${ }^{3}$ Marco Aiello $\left(\mathbb{D},{ }^{3}\right.$ Emanuele Nicolai, ${ }^{3}$ Andrea Soricelli, ${ }^{3,4}$ \\ Marco Giannelli $\left(\mathbb{D},{ }^{5}\right.$ and Stefano Diciotti ${ }^{2}$ \\ 1 "Mario Serio" Department of Experimental and Clinical Biomedical Sciences, University of Florence, Florence, Italy \\ ${ }^{2}$ Department of Electrical, Electronic and Information Engineering "Guglielmo Marconi”, University of Bologna, Bologna, Italy \\ ${ }^{3}$ IRCCS SDN, Naples, Italy \\ ${ }^{4}$ University of Naples Parthenope, Naples, Italy \\ ${ }^{5}$ Unit of Medical Physics, Pisa University Hospital “Azienda Ospedaliero-Universitaria Pisana”, Pisa, Italy
}

Correspondence should be addressed to Marco Giannelli; m.giannelli@ao-pisa.toscana.it

Received 22 April 2020; Accepted 22 April 2020; Published 29 June 2020

Copyright (c) 2020 Mario Mascalchi et al. This is an open access article distributed under the Creative Commons Attribution License, which permits unrestricted use, distribution, and reproduction in any medium, provided the original work is properly cited.

In the article titled "Handedness side and magnetization transfer ratio in the primary sensorimotor cortex central sulcus" [1], affiliation 3 was incorrect. The correct affiliation is "IRCCS SDN, Naples, Italy," and it is corrected above.

\section{References}

[1] M. Mascalchi, S. Ciulli, A. Bianchi et al., "Handedness side and magnetization transfer ratio in the primary sensorimotor cortex central sulcus," BioMed Research International, vol. 2019, Article ID 5610849, 7 pages, 2019. 